\title{
Discontinuation of infliximab after attaining low disease activity in patients with rheumatoid arthritis: RRR (remission induction by Remicade in RA) study
}

\author{
Y Tanaka, ${ }^{1}$ T Takeuchi, ${ }^{2}$ T Mimori, ${ }^{3}$ K Saito, ${ }^{1}$ M Nawata, ${ }^{1}$ H Kameda, ${ }^{4}$ T Nojima, ${ }^{3}$ \\ N Miyasaka, ${ }^{5}$ T Koike ${ }^{6}$; for the RRR study investigators
}

\begin{abstract}
${ }^{1}$ The First Department of Internal Medicine, University of Occupational and Environmental Health, Japan, Kitakyushu, Japan

2Division of Rheumatology and Clinical Immunology, Department of Internal Medicine, Faculty of Medicine, Keio University, Tokyo, Japan ${ }^{3}$ Department of Rheumatology and Clinical Immunology, Kyoto University Graduate School of Medicine, Kyoto, Japan ${ }^{4}$ Department of Rheumatology/ Clinical Immunology, Saitama Medical Centre, Saitama Medical University, Kawagoe, Japan

5Department of Medicine and Rheumatology, Tokyo Medical and Dental University, Tokyo, Japan

${ }^{6}$ Department of Medicine II, Hokkaido University, Sapporo, Japan
\end{abstract}

\section{Correspondence to}

Professor Yoshiya Tanaka,

The First Department of Internal Medicine, School of Medicine, University of Occupational and Environmental Health, Japan, 1-1 Iseigaoka, Kitakyushu 8078555 Japan;

tanaka@med.uoeh-u.ac.jp

Accepted 9 January 2010

\author{
ABSTRACT \\ Background Tumour necrosis factor (TNF) inhibitors \\ enable tight control of disease activity in patients \\ with rheumatoid arthritis (RA). Discontinuation of TNF \\ inhibitors after acquisition of low disease activity (LDA) is \\ important for safety and economic reasons.
}

Objective To determine whether infliximab might be discontinued after achievement of LDA in patients with RA and to evaluate progression of articular destruction during the discontinuation.

Methods 114 patients with RA who had received infliximab treatment, and whose Disease Activity Score, including a 28-joint count (DAS28) was $<3.2$ (LDA) for 24 weeks, were studied.

Results The mean disease duration of the 114 patients was 5.9 years, mean DAS28 5.5 and mean modified total Sharp score (mTSS) 63.3. After maintaining LDA for $>24$ weeks by infliximab treatment, the drug was discontinued and DAS28 in 102 patients was evaluated at year 1. Fifty-six patients (55\%) continued to have DAS $28<3.2$ and $43 \%$ reached DAS $<2.6$ at 1 year after discontinuing infliximab. For 46 patients remission induction by Remicade in RA (RRR) failed: disease in 29 patients flared within 1 year and DAS28 was $>3.2$ at year 1 in 17 patients. Yearly progression of mTSS ( $\Delta$ TSS) remained $<0.5$ in $67 \%$ and $44 \%$ of the RRR-achieved and RRR-failed groups, respectively. The estimated $\Delta \mathrm{mTSS}$ was 0.3 and 1.6 and Health Assessment QuestionnaireDisability Index was 0.174 and 0.614 in the RRR-achieved and RRR-failed groups, respectively, 1 year after the discontinuation.

Conclusion After attaining LDA by infliximab, 56 (55\%) of the 102 patients with RA were able to discontinue infliximab for $>1$ year without progression of radiological articular destruction.

\section{INTRODUCTION}

Rheumatoid arthritis (RA) is a chronic, systemic inflammatory disease that causes significant morbidity and mortality. The combined use of biological agents targeting tumour necrosis factor (TNF) and methotrexate (MTX) has produced significant improvements in clinical, radiographic and functional outcomes that were not previously seen and has revolutionised the treatment goal of RA to clinical remission, structural remission and functional remission. ${ }^{1-5}$ The next goal should be remission without the use of biological agents and subsequent drug-free remission. Although global evidence of the efficacy and safety of TNF inhibitors such as infliximab has accumulated, including the ATTRACT study, ASPIRE study, our RECONFIRM studies and many others, ${ }^{5-10}$ there is no well-established firm evidence for remission free from the use of biological agents.

The initial report of the potential for remission without the use of biological agents in patients with RA was reported by a British group (TNF20 study). ${ }^{11}$ The combination of infliximab and MTX in patients with early RA who had fewer than 12 months of symptoms provided tight control of the disease activity and a significant reduction in MRI evidence of synovitis and erosions at 1 year. At 2 years, functional and quality of life benefits were sustained, despite withdrawal of infliximab treatment. On the other hand, the Behandelstrategieën (BeSt) study was conducted to observe clinical and radiological outcomes of patients with early RA treated with initial infliximab and MTX who discontinued infliximab after achieving a sustained Disease Activity Score $(\mathrm{DAS}) \leq 2.4$. Five years after receiving infliximab and MTX as initial treatment for RA, 58\% of 120 patients discontinued infliximab because of a continuous DAS $\leq 2.4$ and $19 \%$ of patients have stopped all antirheumatic drugs and remain in clinical remission, with minimal joint damage progression. These findings indicate that treatment using infliximab and MTX, guided by DAS, is an effective and tight control to maintain low disease activity (LDA) and may alter the course of early RA. ${ }^{12-16}$

Discontinuation of TNF inhibitors after acquisition of LDA is important for reasons of safety and economy. For instance, the problem of the incidence of haematological malignancy owing to the longterm use of TNF inhibitors remains unresolved. In Japan a large majority of patients have to pay $30 \%$ of their medical costs and all wish to know for how long biological agents must be continued, but we have no answer. We successfully discontinued infliximab after attaining DAS-guided remission for $>24$ weeks, ${ }^{10}$ but evidence based on multicentre studies is needed. Reports published to date on this topic are confined to those from the BeSt study and TNF20 study involving only patients at an early stage of RA. ${ }^{11-16}$

Thus, this multicentre study was undertaken to seek the possibility of discontinuing infliximab after attaining DAS-guided remission and maintaining LDA without infliximab, in patients with RA, including patients with long-established disease, and to evaluate progression of articular destruction and functional disabilities during the discontinuation. online under the BMJ Journals unlocked scheme, see http:// ard.bmi.com/info/unlocked.dtl 


\section{PATIENTS AND METHODS}

\section{Patients}

Data and information on patients with RA fulfilling the diagnostic criteria of the American College of Rheumatology were collected from 26 centres of remission induction by Remicade in RA (RRR) investigator groups in Japan. ${ }^{17}$ Disease activity of individual patients was assessed by Disease Activity Score, including a 28-joint count (DAS28)-erythrocyte sedimentation rate (ESR) or DAS28-C reactive protein (CRP) that was calculated according to the authorised formula (http://www.das-score.nl/, accessed 15 February 2010). ${ }^{18}$ Since none of the patients, except for one, achieved LDA measured by DAS28 despite MTX or a combination of MTX and other disease-modifying antirheumatic drugs for at least 3 months, infliximab treatment $(3 \mathrm{mg} \mathrm{kg}$, every 8 weeks) was administered in the investigators' institutions, according to the treatment guideline proposed by the Japan College of Rheumatology. ${ }^{17}$ Joint damage was assessed by the van der Heijde-modified total Sharp score (mTSS) ${ }^{19}$ and for 102 patients, $\mathrm{x}$-ray images of the hands and feet at baseline, RRR-study entry and 1 year after the study were available; these were evaluable for 49 patients owing to loss of the radiographs and/or low quality of the x-ray images. ${ }^{20} 21$ Two blinded expert readers independently scored articular damage and progression according to the mTSS scoring method. The difference between the two readers' scores for each patient's radiographs was $<1 \%$ of the maximum mTSS score-that is, 448. ${ }^{920} 21$ To confirm that the x-ray results of the 49 patients represented the outcomes of the whole group, we compared multiple background characteristics and changes of each characteristic from baseline to RRRstudy entry between 49 patients with evaluable x-ray images and 53 patients without them and no significant difference was seen between the two groups.

After patients had achieved DAS28 (ESR) $<3.2$ (LDA) for $>24$ weeks, informed consent to discontinue infliximab was obtained from 126 patients. Other criteria were that patients were controlled with $<5 \mathrm{mg} /$ day of oral prednisolone (PSL) and were $>18$ years old. Concomitant use of MTX was started in all patients, and the dose of MTX was determined by each attending doctor. Twelve patients dropped out at the screening period, and 114 patients were enrolled in the study and discontinued infliximab (figure 1). The demographic indicators and baseline disease characteristics of the 114 patients enrolled are summarised in table 1.

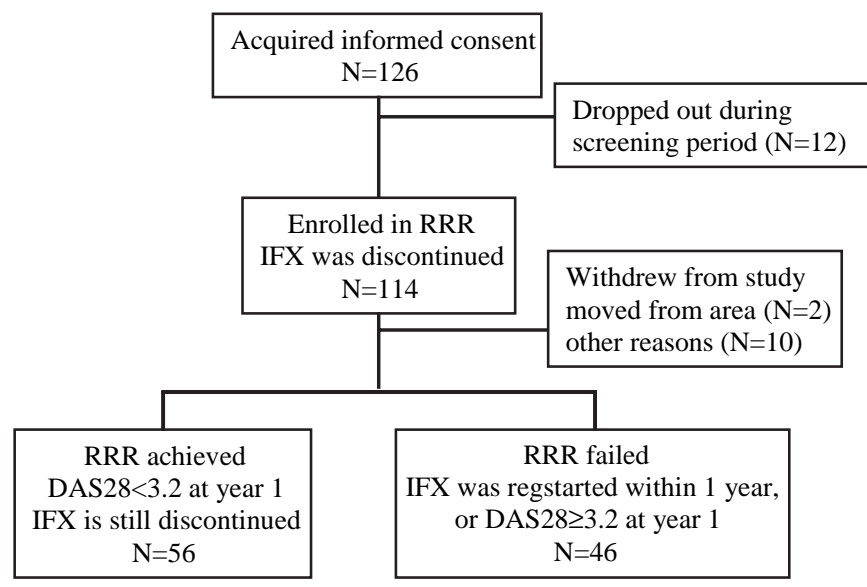

Figure 1 Study design and profile. DAS28, Disease Activity Score, including a 28-joint count; IFX, infliximab; RRR, remission induction by Remicade in rheumatoid arthritis.
Table 1 Demographic indicators and baseline disease characteristics

\begin{tabular}{|c|c|c|c|c|}
\hline & $\begin{array}{l}\text { Enrolled patients } \\
(\mathrm{N}=114)\end{array}$ & $\begin{array}{l}\text { RRR-achieved } \\
(N=56)\end{array}$ & $\begin{array}{l}\text { RRR-failed } \\
(\mathrm{N}=46)\end{array}$ & $\begin{array}{l}\text { p (probability } \\
>\chi^{2} \text { ) }\end{array}$ \\
\hline Women & $87(76 \%)$ & $42(75 \%)$ & $38(83 \%)$ & 0.4691 \\
\hline Age (years) & $51.4(20.0-73.0)$ & $49.5 \pm 12.6$ & $56.1 \pm 12.2$ & 0.0053 \\
\hline $\begin{array}{l}\text { Disease } \\
\text { duration } \\
\text { (years) }\end{array}$ & $5.9(0.1-38.0)$ & $4.8 \pm 5.9$ & $7.8 \pm 7.7$ & 0.0238 \\
\hline $\begin{array}{l}\text { Tender joint } \\
\text { count }(0-28)\end{array}$ & $8.2 \pm 6.7$ & $8.6 \pm 7.0$ & $7.5 \pm 5.8$ & 0.5798 \\
\hline $\begin{array}{l}\text { Swollen } \\
\text { joint count } \\
(0-28)\end{array}$ & $9.0 \pm 7.2$ & $10.1 \pm 7.7$ & $7.6 \pm 5.8$ & 0.1674 \\
\hline $\begin{array}{l}\text { PaGA } \\
\text { (0-100 mm, } \\
\text { VAS) }\end{array}$ & $50.0 \pm 23.0$ & $50.0 \pm 24.2$ & $49.3 \pm 23.1$ & 0.9520 \\
\hline CRP (mg/dl) & $2.5 \pm 3.0$ & $2.6 \pm 2.6$ & $2.7 \pm 3.7$ & 0.5531 \\
\hline ESR $(\mathrm{mm} / \mathrm{h})$ & $46.2 \pm 26.9$ & $43.1 \pm 24.2$ & $54.1 \pm 30.1$ & 0.1555 \\
\hline $\begin{array}{l}\text { DAS28 (ESR) } \\
\text { score }\end{array}$ & $5.5 \pm 1.2$ & $5.5 \pm 1.4$ & $5.6 \pm 1.1$ & 0.9112 \\
\hline $\begin{array}{l}\text { DAS28 (CRP) } \\
\text { score }\end{array}$ & $4.9 \pm 1.2$ & $5.1 \pm 1.3$ & $4.8 \pm 1.3$ & 0.5486 \\
\hline HAQ-DI & $1.0 \pm 0.7$ & $0.9 \pm 0.6$ & $1.2 \pm 0.7$ & 0.1112 \\
\hline mTSS* & $63.3(1.0-314.0)$ & $46.9 \pm 46.5$ & $97.2 \pm 86.9$ & 0.0207 \\
\hline $\mathrm{RF}(\mathrm{U} / \mathrm{ml})$ & $\begin{array}{l}201.9 \pm 496.5 \\
(68.5 \%)\end{array}$ & $225.7 \pm 583.3$ & $197.9 \pm 427.8$ & 0.5190 \\
\hline MTX (mg/week) & $7.7 \pm 2.3$ & $7.9 \pm 1.9$ & $7.8 \pm 2.8$ & 0.3232 \\
\hline PSL (mg/day) & $2.5 \pm 3.4(45.6 \%)$ & $2.4 \pm 3.5$ & $2.8 \pm 3.5$ & 0.5223 \\
\hline
\end{tabular}

Data are number of patients (\%) for categorical data and the means for continuous data. Statistical difference was assessed by non-parametric Wilcoxon $t$ test and $p$ (probability $>\chi^{2}$ ) values are shown. Values in italic indicate a significant difference $(p<0.05)$. *Data supplied for 33 patients who achieved RRR and 16 patients for whom RRR failed. CRP, C-reactive protein; DAS28, Disease Activity Score, including a 28-joint count; ESR, erythrocyte sedimentation rate; HAO-DI, Health Assessment QuestionnaireDisability Index; mTSS, modified total Sharp score; MTX, methotrexate; PaGA, patient global assessment of disease activity; PSL, prednisolone; RF, rheumatoid factor; RRR, remission induction by Remicade in rheumatoid arthritis; VAS, visual analogue scale.

\section{Procedures}

Study protocol was a simple observation after discontinuation of infliximab. The follow-up observation was monitored by symptoms, signs and DAS28 (ESR) every 4-13 weeks for 2 years. The dose of concomitant MTX was basically consistent, but tapering of non-steroidal anti-inflammatory drugs and glucocorticoid was allowed during the study period. The primary end points were that after discontinuing infliximab, DAS28 remains $<3.2$ (LDA) for 1 year and (B) yearly progression of $\mathrm{mTSS}$ remains $<0.5$ (structural remission) for 1 year. Secondary end points were DAS28 remains $<2.6$ (clinical remission) for 1 year, DAS28 remains $<3.2$ for 2 years, yearly progression of mTSS remains $<0.5$ for 2 years and no rescue with infliximab for 1 or 2 years is needed, after discontinuing infliximab. When a flare-up occurred in patients after the discontinuation, restart of infliximab was allowed and patients were categorised into the 'RRR-failed' group. For the restart of infliximab, the same dose $(3 \mathrm{mg} / \mathrm{kg})$ and the same premedication as used before the study entry were used.

\section{Statistical analysis}

Baseline characteristics of patients are summarised in table 1 using the mean values for continuous variables. All multivariate analyses were conducted using the variables gender, age, duration of disease, DAS28 (ESR) score, DAS28 (CRP) score, tender joint count (0-28), swollen joint count (0-28), patient global assessment of disease activity ( $\mathrm{PaGA}, 0-100 \mathrm{~mm}$, visual analogue scale), ESR, CRP, Health Assessment Questionnaire-Disability Index (HAQ-DI), rheumatoid factor (RF), MTX dose and PSL dose at baseline. Spearman correlation analyses were performed to evaluate the 
association between multivariables at RRR-study entry and DAS28 at the primary end point (last observation carried forward) of 102 patients. Logistic regression analysis was carried out to estimate DAS28 at the primary end point as dependent variables (probability) by DAS28 at RRR entry as independent variables. A receiver operating characteristic (ROC) curve was developed based on the logistic analysis and the significant cut-off point was determined from the curve. For categorical response parameters, group comparisons were made using a non-parametric Wilcoxon t test. Statistical analyses were performed using JMP software version 7 (SAS Institute, Cary, North Carolina, USA). All reported p values are two sided and $p$ values $<0.05$ were considered significant.

\section{RESULTS}

\section{Study end points}

The demographic indicators and baseline characteristics of the 114 patients enrolled were as follows: mean age 51.4 years, mean disease duration 5.9 years and mean mTSS 63.3, indicating that the population included patients with long-established disease, and the mean DAS28 (ESR) score was 5.5, implying that most patients had highly active disease (table 1). Figure 1 shows the study profile. After maintaining DAS28<3.2 (LDA) for $>24$ weeks by infliximab treatment, infliximab was discontinued in 114 patients. Twelve patients withdrew because they moved from area $(n=2)$ and for other reasons $(n=10)$, and thus DAS28 could be evaluated in 102 patients at year 1 .

Of the 102 patients, 56 patients achieved the primary end point having a DAS28<3.2 and remaining without infliximab for 1 year after the discontinuation (figure $2 \mathrm{~A}$ ). Thus, $55 \%$ of the enrolled patients met the primary end point that LDA was maintained for 1 year after discontinuing infliximab. Furthermore, 44 patients $(43 \%)$ reached $\mathrm{DAS}<2.6$ after the discontinuation. On the other hand, 29 patients flared within 1 year (mean duration was 6.4 months) after the discontinuation and in 17 patients DAS28 was $>3.2$ at year 1 and thus RRR failed for 46 patients $(45 \%)$ at year 1 . Re-treatment with infliximab in 32 patients was effective and the majority of patients reached DAS28<3.2 within 24 weeks (figure 2B). Minimal adverse reactions at infusion of the agent were seen in five patients only at the first or second infusion.

To clarify the background factors related to the RRRachievement, multiple clinical parameters at baseline were compared between patients for whom RRR was achieved and those for whom it failed. Patients for whom RRR was achieved were younger (49.5 vs 56.1), their disease duration was shorter (4.8 vs 7.8) and mTSS was lower (46.9 vs 97.2) than for those for whom RRR failed. Among 56 patients who achieved RRR, 10 patients had early RA (disease duration $<1.0$ year) and eight long-established disease ( $>10$ years). Of 46 patients for whom RRR failed, eight had early RA and 12 established disease. These results imply that infliximab can be discontinued in patients with long-established RA. In contrast, no significant difference was seen in gender, DAS including DAS28, tender or swollen joint count, ESR and CRP, HAQ-DI, RF and the dose of MTX and PSL. Since these factors interact with one another, we analysed the relationship between RRR-achievement and a series of clinical parameters at baseline using multivariate analysis after adjusting for confounding variables. No significant relations
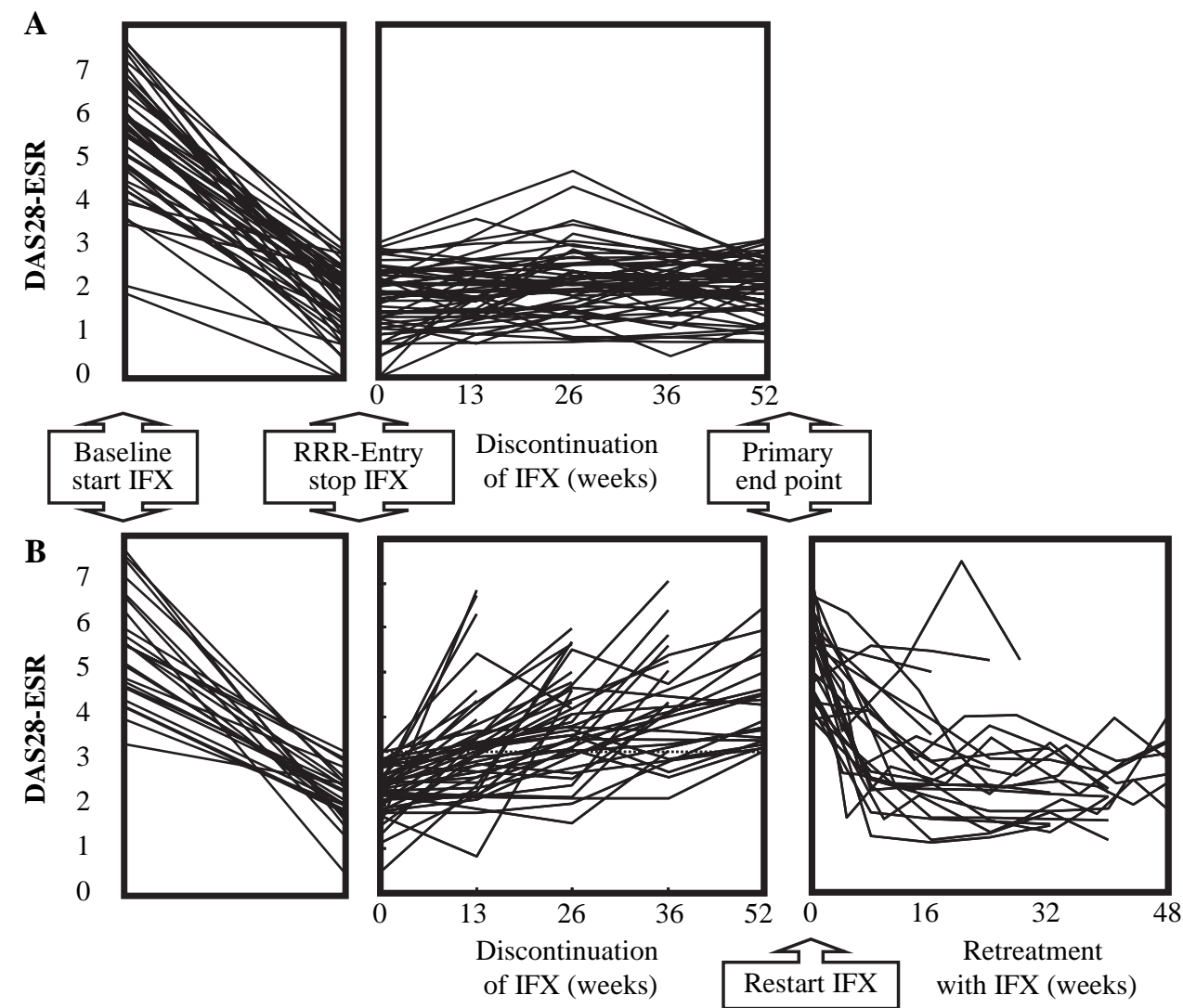

Figure 2 Changes of Disease Activity Score, including a 28-joint count (DAS28) in patients with remission induction by Remicade in rheumatoid arthritis-achieved (RRR-achieved) and patients for whom RRR failed (RRR-failed). (A) Changes of Disease Activity Score, including a 28-joint count (DAS28) at baseline when infliximab (IFX) was administered, at RRR-study entry when infliximab was discontinued and at the primary end point at week 52 after discontinuing IFX in 56 patients who were still satisfied with DAS28 (erythrocyte sedimentation rate (ESR)) $<3.2$ at week 52 , RRRachieved'. (B) Changes of DAS28 at baseline, at RRR entry and the end point in 46 patients whose disease activity flared after the discontinuation of IFX or DAS28 > 3.2 at week 52, 'RRR-failed'. The lower right panel shows changes of DAS28 after the restarting IFX in 32 patients for whom RRR failed. 
between RRR-achievement and age, gender, DAS28 (ESR) score, $\mathrm{PaGA}$ and CRP were found, whereas a significant correlation was found with disease duration $(p=0.0019)$ and serum levels of $R F(p=0.0128)$ in RRR-achievement.

To determine the correlation of DAS28 at the primary end point with clinical parameters at RRR-study entry, univariate analysis of multiple variables was carried out. No significant correlations between DAS28 (ESR) at the primary end point and a series of clinical parameters were found, whereas DAS28 (ESR) and DAS28 (CRP) at RRR-entry were significantly correlated with DAS28 (ESR) at the primary end point. Subsequently, logistic regression analysis to estimate the probability of DAS2 $8<3.2$ at the primary end point as dependent variable by DAS28 at RRR-entry as independent variable was assessed. A significant logistic regression curve was drawn between the dependent and independent variables $(p=0.0005)$ (figure 3A). Thus, DAS28 at RRR-study entry had the most marked correlation with the maintenance of LDA for 1 year after the discontinuation. By reciprocal statistics, DAS28 at RRR-study entry was estimated as 2.22 , to attain DAS28<3.2 at the end point in $50 \%$ of the 102 patients (figure $3 \mathrm{~A}$ ). Furthermore, $71.4 \%$ of patients whose DAS28 at study entry was $<2.225$, a cut-off point calculated from the ROC curve, continued to have DAS28<3.2 for 1 year, whereas only $32.6 \%$ of patients whose DAS28 at RRR-entry was 2.225-3.2 continued to have DAS28<3.2 (figure 3B), indicating that 'deep remission' was required to maintain lower disease activity for 1 year after discontinuation of infliximab.

\section{Structural and functional changes}

From the 102 patients enrolled in the study, 49 patients were selected in whom both hand and feet x-ray data were available and evaluable; experts examined the structural damage before and after the infliximab treatment. When the baseline characteristics of the 49 patients in the study were compared with the rest of the patients in the study with insufficient $x$-ray data $(n=53)$, no significant difference was seen. Next, the baseline characteristics of the 33 patients who achieved RRR and 16 patients for whom RRR failed were compared. As described in table 1, disease duration was shorter and mTSS was lower in patients who achieved RRR than in patients for whom RRR failed, but yearly progression of mTSS ( $\Delta$ mTSS) was comparable between two groups (table 2 ). $\Delta$ mTSS at RRR entry was also comparable between two groups. However, means (0.3 vs 1.6) and medians ( 0.0 vs 1.5 ) of $\Delta$ mTSS were lower in the RRRachieved group than in the RRR-failed group and more patients in the RRR-achieved group (67\%) achieved $\Delta \mathrm{mTSS}<0.5$, radiographic remission, than patients in the RRR-failed group (44\%). Thus, another primary end point for structural remission was achieved for 1 year after the discontinuation. Furthermore, HAQ-DI at baseline and RRR entry was comparable between patients for whom RRR was achieved and those for whom it failed, whereas HAQ-DI at the primary end point in patients who achieved RRR was significantly lower than that in patients for whom RRR failed (0.174 vs 0.614) (figure 4).

\section{DISCUSSION}

This multicentre study was undertaken to determine the possibility of discontinuing infliximab treatment in patients with RA after acquiring DAS-guided LDA, including those with longestablished disease. Among 102 patients who could be evaluated at year 1, 56 patients (55\%) satisfied the primary end point by maintaining DAS28<3.2 (LDA) and 44 patients (43\%) reached $\mathrm{DAS}<2.6$ (remission), remaining without infliximab at year 1 after the discontinuation. Of the 102 patients, 83 (81.4\%) were in clinical remission at study entry and after discontinuing infliximab, 39/83 patients (47\%) remained in remission and 10/83 patients $(12 \%)$ progressed to LDA at the primary end point.

These data are similar to those of the BeSt study. However, the greatest difference between the patient populations enrolled in the two studies is mean disease duration-0.4 years in the BeSt study versus 5.9 years in our RRR study. ${ }^{12-16}$ Joint destruction also differed between the two studies-mean mTSS 7.0 in the BeSt study versus 63.3 in our RRR study-suggesting that discontinuation of infliximab after reaching LDA is possible in patients with early RA and also in patients with long-established disease. ${ }^{13-15}$ On the other hand, among multiple clinical parameters at baseline, disease duration was statistically related to RRR-achievement by multivariate analysis and disease duration was shorter (4.8 vs 7.8) and mTSS was lower (46.9 vs 97.2) in patients who achieved
A

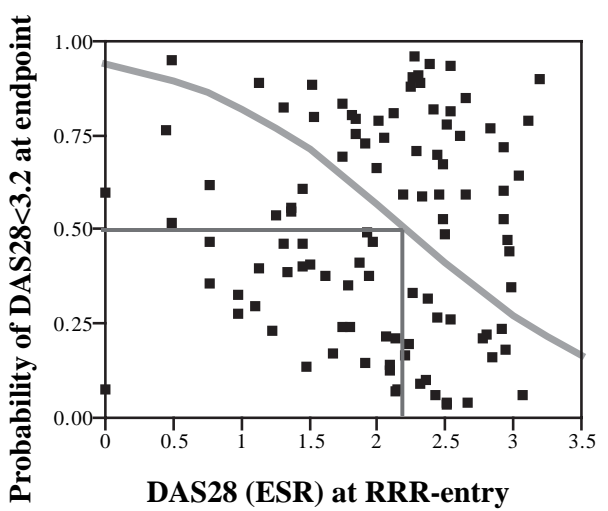

B

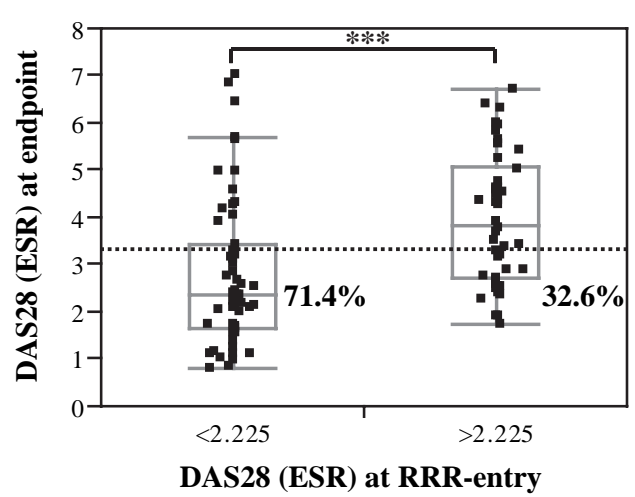

Figure 3 Logistic analysis of probability of Disease Activity Score, including a 28-joint count (DAS28) was $<3.2$ at primary end point by DAS28 at remission induction by Remicade in rheumatoid arthritis entry (RRR entry). (A) Logistic regression analysis to estimate DAS28 at primary end point as dependent variables by DAS28 at RRR entry as independent variables. The y-axis shows the probability of DAS28 $<3.2$ at the primary end point after the 52 weeks discontinuation of infliximab and a scatter diagram of an individual patient and logistic regression curve (solid line) are shown. To attain DAS28 $<3.2$ at the end point in $50 \%$ of the 102 patients, DAS28 at RRR study entry was estimated by reciprocal statistics. (B) From the receiver operating characteristic curve based on the logistic regression analysis above, the cut-off point of DAS28 at RRR-study entry was 2.225 . Subsequently, one-way analysis of DAS28 at the primary end point by DAS28 at study entry, $<2.225$ versus between 2.225 and 3.2 , was performed and the statistical difference of the two groups was sought by non-parametric Wilcoxon t test $\left({ }^{* *} p<0.001\right)$. ESR, erythrocyte sedimentation rate. 
Table 2 Radiographic indicators and baseline disease characteristics

\begin{tabular}{|c|c|c|c|}
\hline & $\begin{array}{l}\text { RRR-achieved } \\
(\mathrm{N}=33)\end{array}$ & $\begin{array}{l}\text { RRR-failed } \\
(\mathrm{N}=16)\end{array}$ & $\begin{array}{l}\text { p (probability } \\
\left.>\chi^{2}\right)\end{array}$ \\
\hline \multicolumn{4}{|l|}{ Baseline } \\
\hline $\begin{array}{l}\text { Disease duration } \\
\text { (years) }\end{array}$ & $4.7(0.5-14.0)$ & $8.6(0.5-25.0)$ & 0.0280 \\
\hline DAS28 (ESR) score & $5.5(1.9-7.6)$ & $5.7(4.2-6.8)$ & 0.6976 \\
\hline HAQ-DI & $1.0(0.0-2.3)$ & $1.1(0.0-1.8)$ & 0.6271 \\
\hline mTSS & $46.9(1.0-216.5)$ & $97.2(6.0-314.0)$ & 0.0207 \\
\hline Bone erosion score & $23.7(0.0-127.5)$ & $55.5(1.5-192.5)$ & 0.0119 \\
\hline $\begin{array}{l}\text { Joint space } \\
\text { narrowing score }\end{array}$ & $23.2(1.0-89.0)$ & $41.6(4.5-121.5)$ & 0.0621 \\
\hline $\begin{array}{l}\text { Yearly progression } \\
\text { of mTSS }\end{array}$ & $13.1(0.8-51.3)$ & $15.0(1.0-47.8)$ & 0.5794 \\
\hline \multicolumn{4}{|l|}{ RRR-entry } \\
\hline $\begin{array}{l}\text { Yearly progression } \\
\text { of mTSS }\end{array}$ & $1.0(-2.9$ to 10.5$)$ & $0.7(-2.0$ to 6.7$)$ & 0.5788 \\
\hline \multicolumn{4}{|l|}{ Primary end point } \\
\hline $\begin{array}{l}\text { Yearly progression } \\
\text { of mTSS }\end{array}$ & $0.3(-3.6$ to 8.5$)$ & $1.6(-3.6$ to 7.0$)$ & 0.1087 \\
\hline $\begin{array}{l}\text { Median of yearly } \\
\text { progression of } \\
\text { mTSS }\end{array}$ & 0.0 & 1.5 & - \\
\hline $\begin{array}{l}\text { Yearly progression } \\
\text { of mTSS }<0.5(\%)\end{array}$ & 67 & 44 & 0.2161 \\
\hline
\end{tabular}

Data are number of patients (\%) for categorical data and the means for continuous data. Statistical difference was assessed by non-parametric Wilcoxon $t$ test and $p$ (probability $>\chi^{2}$ ) values were shown. Values in italic indicate a significant difference $(p<0.05)$. DAS28, Disease Activity Score based on assessments of 28 tender and 28 swollen joints; ESR, erythrocyte sedimentation rate; HAQ-DI, Health Assessment QuestionnaireDisability Index; mTSS, modified total Sharp score; RRR, remission induction by Remicade in rheumatoid arthritis.

RRR than in those for whom RRR failed. These results imply that remission free from biological agents can be more easily obtained in patients with shorter disease duration than in those with more established disease, but discontinuation of infliximab is still possible even in patients with long-established RA, because eight patients whose disease duration was $>10$ years successfully remained without infliximab for 1 year.

Among 102 evaluated patients, disease in 29 patients flared within 1 year, 17 patients had DAS28 $\geq 3.2$ at year 1 after discontinuing infliximab and 32 patients had already been re-treated with infliximab. One of the major concerns of restarting infliximab is the possibility of an infusion reaction after the longterm discontinuation, partly owing to human anti-chimeric antibodies. ${ }^{22}$ However, minimal adverse reactions at infusion of the agent were seen only in five patients at the first or second infusion. Another concern is the progress of joint damage after discontinuation of infliximab. However, although the yearly progression of mTSS at RRR-study entry was also comparable between two groups, means ( 0.3 vs 1.6) and medians ( 0.0 vs 1.5 ) of $\triangle$ mTSS were shorter in the RRR-achieved group than in the RRR-failed group. Furthermore, at year 1 after the discontinuation of infliximab, more patients in the RRR-achieved group $(67 \%)$ tended to satisfy $\Delta \mathrm{mTSS}<0.5$ - that is, structural remission, than those in the RRR-failed group (44\%) and HAQ-DI in patients who achieved RRR was significantly lower than that in patients for whom RRR failed ( 0.174 vs 0.614$)$. These results indicate that both structural remission and functional remission were maintained for 1 year in patients with LDA even after discontinuing infliximab.

This study also shows the significance of DAS-guided tight control of RA in order to maintain remission free from biological agents. There was a significant correlation between DAS28 (ESR) or DAS28 (CRP) at RRR entry and DAS28 (ESR) at year 1 after the discontinuation of infliximab by univariate analysis of multiple variables and a logistic regression analysis. Thus, DAS28 at RRR-study entry had the greatest correlation with maintenance of LDA for 1 year after discontinuation. Also, DAS28 at study entry was mainly influenced by PaGA and ESR among the composite measures. By reciprocal statistics, the estimated DAS28 (ESR) at RRR-study entry was $2.22(1.85-2.70)$, to attain DAS28<3.2 at the primary end point in $50 \%$ of the 102 patients studied. Also, $71.4 \%$ of patients whose DAS28 at study entry was $<2.225$, a cut-off point calculated from ROC curve, remained DAS28<3.2 for 1 year, whereas only $32.6 \%$ of patients whose DAS28 at RRR-entry was 2.225-3.2 remained DAS28<3.2. These results indicate that 'deep remission' appears to be required to maintain lower disease activity for 1 year after discontinuation of infliximab.

About $55 \%$ of the 102 patients, who were in an LDA state for $>24$ weeks with infliximab and MTX treatment, could discontinue infliximab for $>1$ year without progression of radiological articular destruction or functional disturbance. These data may have significant implications for the optimal use of expensive biological treatments: (a) re-treatment with infliximab is efficient and tolerable in the patients for whom RRR failed; (b) DASguided monitoring is valuable to keep tight control; $(c)$ 'deep

A

Baseline

B

At RRR entry

C At primary endpoint

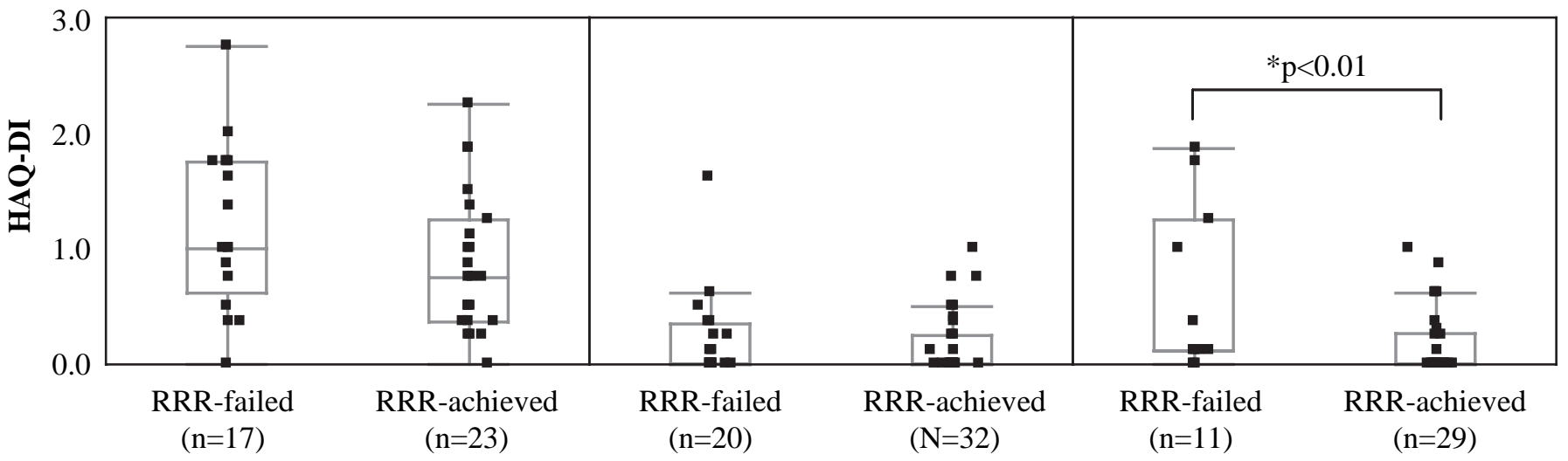

Figure 4 Health Assessment Questionnaire-Disability Index (HAQ-DI) in patients for whom remission induction by Remicade in rheumatoid arthritis failed (RRR-failed)' and in patients for whom 'RRR was achieved (RRR-achieved)' at (A) baseline, (B) RRR entry and (C) the primary end point. The line in the box represents the median value and the upper and lower ends of the box indicate the 25th and 75th centiles of the population. Statistical difference was assessed by non-parametric Wilcoxon t test. 
remission' by tight control is required to maintain discontinuation of infliximab; (d) remission free from biological agents may be easier to attain in patients with early RA, but is possible for patients with long-established disease; (e) treatment aimed at reaching a target of $\mathrm{LDA}$ is pivotal to the approach to remission free from biological agents. Finally, TNF $\alpha$ is not a cause of RA, but if appropriate treatment with infliximab can lead to drug-free remission, TNF inhibitors may shut down pathological processes and may change or modify the disease course in RA. Thus, a clinical and basic research approach to the "processdriven disease' of RA is warranted.

Acknowledgements We thank all medical staff in all institutions for providing the data.

Funding Supported in part by a research grant-in-aid for scientific research by the Ministry of Health, Labour and Welfare of Japan, the Ministry of Education, Culture, Sports, Science and Technology of Japan and the University of Occupational and Environmental Health, Japan.

Competing interests YT has received consultant fees from MitsubishiTanabe Pharma, Pfizer Inc; lecture fees from Mitsubishi-Tanabe Pharma, Takeda Pharmaceutical Co Ltd, Abbott, Eisai Pharma, Chugai Pharma. TT has received consultant fees from Mitsubishi-Tanabe Pharma, Wyeth Japan, Abbott, Eisai Pharma, Janssen Pharma, Chugai Pharma, Bristol-Myers-Squibb, Novartis; lecture fees from Mitsubishi-Tanabe Pharma, Takeda Pharmaceutical Co Ltd, Abbott, Eisai Pharma, Chugai Pharma. HK has received lecture fees from Mitsubishi-Tanabe Pharma, Centocor, Wyeth Japan, Takeda Pharmaceutical Co Ltd, Abbott, Eisai Pharma, Chugai Pharma. NM has received consultant fees from Mitsubishi-Tanabe Pharma; Abbott, Eisai Pharma, Janssen Pharma, Chugai Pharma, Bristol-Myers-Squibb; lecture fees from Mitsubishi-Tanabe Pharma, Takeda Pharmaceutical Co Ltd, Wyeth Japan, Abbott, Eisai Pharma, Chugai Pharma. TK has received consultant fees from Bristol-MyersSquibb, Abbott; lecture fees from Mitsubishi-Tanabe Pharma, Takeda Pharmaceutical Co Ltd, Wyeth Japan, Abbott, Eisai Pharma, Chugai Pharma.

\section{Patient consent Obtained.}

Ethics approval This study is an observational study and is registered with the University Hospital Medical Information Network-Clinical trials Registry, number R000002571. Also, ethics committees of the participating centres approved the study protocol.

\section{Provenance and peer review Not commissioned; externally peer reviewed.}

RRR study investigators (other than the authors) Shunsuke Fukuyo (University of Occupational and Environmental Health, School of Medicine, Kitakyushu, Japan), Hayato Nagasawa (Saitama Medical Centre, Saitama Medical University, Kawagoe, Japan), Yukitaka Ueki (Sasebo Central Hospital, Sasebo, Japan), Hideo Ohstubo (Japanese Red Cross Kagoshima Hospital, Kagoshima, Japan), Kosaku Murakami (Kyoto University, Kyoto, Japan), Hiroaki Dobashi (Kagawa University, Kagawa, Japan), Shigeru Honjo (Saiseikai Takaoka Hospital, Takaoka, Japan), Teruhisa Azuma (Tenri Hospital), Masato Yagita, Saori Hatachi and Kazuyasu Ushio (Kitano Hospital, Tazuke Kofukai Medical Resarch Institute and Ushio clinic, Osaka, Japan), Toshihide Mimura and Yuji Akiyama (Saitama Medical University, Saitama, Japan), Hiromitsu Takemori (Aomori Prefectural Hospital), Takao Takeuchi (Osaka Red Cross Hospital, Hayaishi Hospital, Osaka, Japan),Tsuyoshi Kasama (Showa University, Tokyo, Japan), Shunsuke Mori (Kumamoto Saishunsou National Hospital, Kumamoto, Japan), Shouhei Nagaoka (Yokohama Minami Kyousai Hospital, Yokohama, Japan), Masaaki Inaba and Hitoshi Goto (Osaka City University, Osaka, Japan), Toshihiko Hidaka (Zenjinkai Shimin-no-Mori Hospital, Miyazaki, Japan), Yasuaki Okuda (Dohgo Spa Hospital, Matsuyama, Japan), Yoshinari Takasaki and Naoto Tamura (Juntendo University, Tokyo, Japan), Kazuhide Tanimura (Tokeidai Memorial Hospital, Sapporo, Japan), Takayuki Sumida (University of Tsukuba, Tsukuba, Japan), Katsumi Eguchi (Nagasaki University, Nagasaki, Japan), Yho Ishiguro (Hirosaki University, Horosaki, Japan), Takeo Sakurai (Inoue Hospital, Gunma, Japan).

\section{REFERENCES}

1. Klareskog L, Catrina Al, Paget S. Rheumatoid arthritis. Lancet 2009;373:659-72.

2. Emery P, Breedveld FC, Hall S, et al. Comparison of methotrexate monotherapy with a combination of methotrexate and etanercept in active, early, moderate to severe rheumatoid arthritis (COMET): a randomised, double-blind, parallel treatment trial. Lancet 2008;372:375-82.

3. Breedveld FC, Weisman MH, Kavanaugh AF, et al. The PREMIER study: a multicenter, randomized, double-blind clinical trial of combination therapy with adalimumab plus methotrexate versus methotrexate alone or adalimumab alone in patients with early, aggressive rheumatoid arthritis who had not had previous methotrexate treatment. Arthritis Rheum 2006;54:26-37.

4. Klareskog L, van der Heijde D, de Jager JP, et al. Therapeutic effect of the combination of etanercept and methotrexate compared with each treatment alone in patients with rheumatoid arthritis: double-blind randomised controlled trial. Lancet 2004;363:675-81.

5. St Clair EW, van der Heijde DM, Smolen JS, et al. For the Active-Controlled Study of Patients Receiving Infliximab for the Treatment of Rheumatoid Arthritis of Early Onset Study Group. Combination of infliximab and methotrexate therapy for early rheumatoid arthritis: a randomized, controlled trial. Arthritis Rheum 2004;50:3432-43.

6. Lipsky PE, van der Heijde DM, St Clair EW, et al. For the Anti-Tumor Necrosis Factor Trial in Rheumatoid Arthritis with Concomitant Therapy Study Group. Infliximab and methotrexate in the treatment of rheumatoid arthritis. N Engl J Med 2000;343:1594-602.

7. Yamanaka H, Tanaka Y, Sekiguchi N, et al. Retrospective clinical study on the notable efficacy and related factors of infliximab therapy in a rheumatoid arthritis management group in Japan (RECONFIRM). Mod Rheumatol 2007:17:28-32.

8. Tanaka Y, Takeuchi T, Inoue E, et al. Retrospective clinical study on the notable efficacy and related factors of infliximab therapy in a rheumatoid arthritis management group in Japan: 1-year clinical and ragiographic outcomes (RECONFIRM-II). Mod Rheumatol 2008;18:146-52.

9. Takeuchi T, Yamanaka H, Inoue E, et al. Retrospective clinical study on the notable efficacy and related factors of infliximab therapy in a rheumatoid arthritis management group in Japan: one-year outcome of joint destruction (RECONFIRM-2J). Mod Rheumatol 2008;18:447-54.

10. Nawata M, Saito K, Nakayamada S, et al. Discontinuation of infliximab in rheumatoid arthritis patients in clinical remission. Mod Rheumatol 2008;18:460-4.

11. Quinn MA, Conaghan PG, O'Connor PJ, et al. Very early treatment with infliximab in addition to methotrexate in early, poor-prognosis rheumatoid arthritis reduces magnetic resonance imaging evidence of synovitis and damage, with sustained benefit after infliximab withdrawal: results from a twelve-month randomized, doubleblind, placebo-controlled trial. Arthritis Rheum 2005;52:27-35.

12. Goekoop-Ruiterman YP, Vries-Bouwstra JK, Allaart CF, et al. Comparison of treatment strategies in early rheumatoid arthritis: a randomized controlled trial. Ann Intern Med 2007; 146:406-15.

13. van der Kooij SM, le Cessie S, Goekoop-Ruiterman YP, et al. Clinical and radiological efficacy of initial vs delayed treatment with infliximab plus methotrexate in patients with early rheumatoid arthritis. Ann Rheum Dis 2009;68:1153-8.

14. Goekoop-Ruiterman YP, de Vries-Bouwstra JK, Allaart CF, et al. Clinical and radiographic outcomes of four different treatment strategies in patients with early rheumatoid arthritis (the BeSt study): a randomized, controlled trial. Arthritis Rheum 2008;58:S126-35.

15. van der Bijl AE, Goekoop-Ruiterman YP, de Vries-Bouwstra JK, et al. Infliximab and methotrexate as induction therapy in patients with early rheumatoid arthritis. Arthritis Rheum 2007:56:2129-34.

16. Goekoop-Ruiterman YP, de Vries-Bouwstra JK, Kerstens PJ, et al. DAS-driven therapy versus routine care in patients with recent-onset active rheumatoid arthritis. Ann Rheum Dis 2010;69:65-9.

17. Miyasaka N, Takeuchi T, Eguchi K. Proposed (corrected) Japanese guidelines for the use of infliximab for rheumatoid arthritis. Mod Rheumato/ 2005;15:4-8.

18. Arnett FC, Edworthy SM, Bloch DA, et al. The American Rheumatism Association 1987 revised criteria for the classification of rheumatoid arthritis. Arthritis Rheum 1988;31:315-24.

19. van der Heijde DMPM, van 't Hof MA, van Riel PLCM, et al. A comparison of validity of single variables and composed indices for measuring disease activity in rheumatoid arthritis. Ann Rheum Dis 1992;51:177-81.

20. van der Heijde D. Radiographic progression in rheumatoid arthritis: does it reflect outcome? Does it reflect treatment? Ann Rheum Dis 2001;60(Suppl 3):iii47-50.

21. van der Heijde D. How to read radiographs according to the Sharp/van der Heijde method. J Rheumatol 2000;27:261-3.

22. Takeuchi T, Tatsuki Y, Nogami Y, et al. Postmarketing surveillance of the safety profile of infliximab in 5000 Japanese patients with rheumatoid arthritis. Ann Rheum Dis 2008;67:189-94. 